Communications in Physics, Vol. 24, No. 3 (2014), pp. 239-245

DOI:10.15625/0868-3166/24/3/3948

\title{
ROLE OF CALCULATION MODEL IN EXPERIMENTALLY DETERMINING THERMAL ENERGY GENERATED IN LASER ROD OF SOLID LASER
}

\author{
PHAM VU THINH AND LE NGOC ANH \\ Institute of Technical Physics, Military Institute of Science and Technology \\ E-mail: ngocanhle74@gmail.com
}

Received 25 April 2014

Accepted for publication 26 Agust 2014

\begin{abstract}
In many solid-state laser pumped by flash lamps, thermal source distributed in laser rod is heterogeneous, complicated and cannot be described by explicit functions. Pumping process can be considered an adiabatic process. Thermal gradient generated within the laser rod is the result of the inhomogeneous distribution of absorbed energy. Calculation model and numerical method can help to determine the relation between the experimental values and thermal energy generated in laser rod.
\end{abstract}

Keywords: solid-state laser, lase rod.

\section{INTRODUCTION}

In a solid-state laser, a significant fraction of absorbed pump radiation is converted into heat instead of contributing to laser radiation [1]. In flashlamp-pumped system, the broad spectral distribution of the pump source causes a significant amount of generated heat. For high repetition rate and high power lasers, thermal effects should be mitigated because these effects may reduce remarkably laser beam quality, interfere with efficient laser operations. Thermal stresses may cause some thermal fracture damages on the laser rod. For this reason, one of the most critical problems in designing, manufacturing a high repetition rate and high power laser system is to address heat dissipation issue. In order to solve the thermo physics problem of the laser rod, we need to determine the generated thermal energy and its distribution within pumped laser rod.

\section{EXPERIMENTAL CAPABILITIES}

Direct measurement of generated thermal energy in laser rod is a complicated problem and technically unfeasible, especially when the pulse width is within the range from a few dozen to hundreds of $\mu \mathrm{s}$, even for flashlamp-pumped system. Therefore, in most cases, its thermal effects determine the thermal energy indirectly.

There are many techniques to evaluate the generated thermal effects in laser rod. Among those, the simplest method is the technique using a probe laser beam [2]. The principle of this method is to measure and determine the variation in the parameters of output probe beam after 
it travels through pumped active medium. Based on caustic changes, we can determine the focal length of the thermal lens. Probe beam technique provides a simple but lack of sufficient accuracy method. Among other techniques, interferometric technique $[3,4]$ provides results with much higher precision. Based on provided interference patterns, we can determine wavefront distortion after the beam travels through the laser rod. However, this method has the disadvantage of intricate experimental configurations and instabilities due to mechanical vibrations. The wavefront sensor method (Shack-Hartmann) is considered to be of adequate accuracy compared to interferometric method while it requires a much simpler experimental configuration and the results obtained are less sensitive to mechanical vibrations [5-7]. With commercial wavefront sensors, we often acquire the accuracy within the range of $\lambda / 15 \div \lambda / 20$. Shack-Hartmann wavefront sensors system in compact form including specialized camera and compatible software helps to ease the measurement of changes of wavefront.

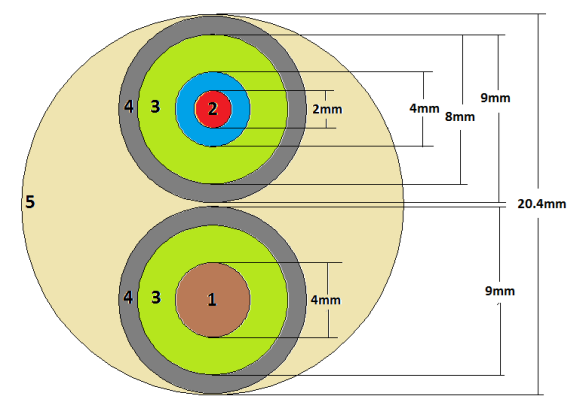

Fig. 1. Schematic diagram of Nd:YAG laser quantron cross section. 1. Laser rod; 2. Flash lamp; 3. Cooling liquid; 4. Cooling liquid tube; 5. Reflector chamber.

Our objective is to investigate the quantron of flashlamp-pumped Nd:YAG solid laser. Fig. 1 illustrates the cross section of this quantron. We use a Nd:YAG laser rod of 1.0 at.\%. The surfaces of the two rod's ends are covered with anti-reflective coating. The scattering surrounding surface is about $20 \%$ to avoid parasitic modes. The length of the laser rod is $50.0 \mathrm{~mm}$. The flash lamp used is of type Xe 450 torr. The light bulb is made of fused quartz and the distance between the two electrodes is $50.0 \mathrm{~mm}$. Water is used as cooling liquid and the routing tube is made of pyrex glass. The reflector chamber is $50.0 \mathrm{~mm}$ long, the inner surface is covered by metal coating with reflective index of $90 \%$. The experimental results based on caustic parameters of the probe beam are presented in details in [2].

We use the Shack-Hartmann wavefront sensor produced by Thorlab, including standardized microlens array, CCD camera, compatible software in our experiments. The microlens array is of type WFS $150-5 \mathrm{C}$, diameter of microlens is of $146.0 \mu \mathrm{m}$, distance between two adjacent microlens center is $150.0 \mu \mathrm{m}$, focal length is $3.7 \mathrm{~mm}$. These microlenses are made of fused quartz. The resolution of the CCD camera is $1280 \times 1024$ pixel, the size of each pixel is $4.65 \mu \mathrm{m} \times 4.65 \mu \mathrm{m}$, the size of the effective sensor is $5.95 \mathrm{~mm} \times 4.76 \mathrm{~mm}$. The number of focus points on microlens array is $29 \times 29$ when the size of region of interest is $4 \mathrm{~mm} \times 4 \mathrm{~mm}$. The precision range of the sensor is $\lambda / 15$. CCD camera can be triggered synchronically with pumping pulses. 
Because the diameter of the laser rod is $4.0 \mathrm{~mm}$, it does not require additional optical system to rebuild images on the sensors. A HeNe laser source with suitable optical system provides collimated beam. Thorlab's sensors can set any wavefront to be the benchmark to evaluate variation which may occur. We set the wavefront of the input collimated beam before it travels into the laser rod to be the benchmark. The specialized software allows to save the measured results in excel files. Fig. 2 and Fig. 3 demonstrate one of our measured results.

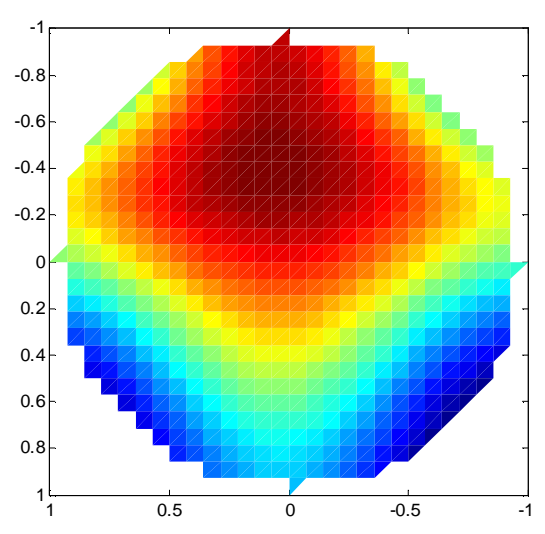

(a) Wavefront

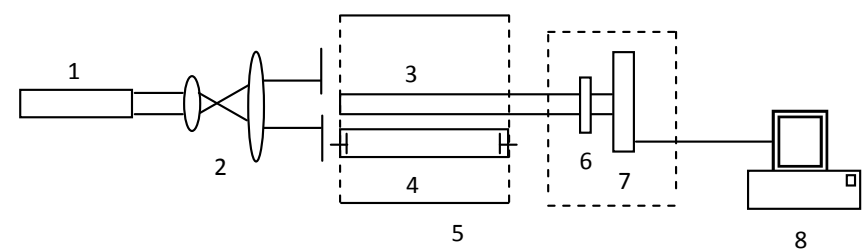

Fig. 2. Experimental diagram of a system using Shack-Hartmann wavefront sensor for measurement of thermal lens effect in solidstate laser rod. 1. He-Ne laser $\lambda=0,6328 \mu m$; 2. Telescope optical system; 3. Laser rod; 4. Flashlamp; 5. Reflective chamber; 6. Microlens array; 7. CCD Camera; 8. Computer.

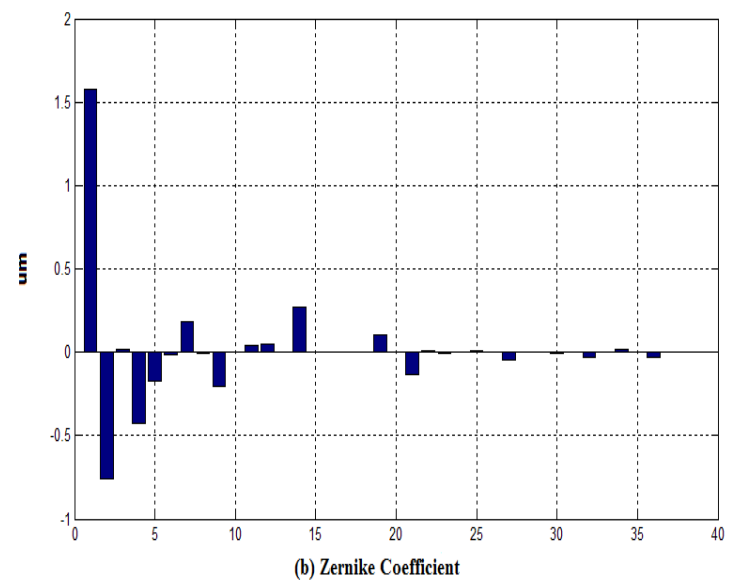

Fig. 3. The measured result of wavefront (a) and Zernike coefficient (b) obtained by using wavefront sensor.

The measured results of Zernike coefficients (Fig. 3b) proved that thermal effects in laser rod not only acts as a convex lens but also cause many other optical aberrations and its contributions to wavefront distortion are significant.

\section{ROLE OF MATHEMATICAL MODEL IN PROCESSING EXPERIMENTAL RESULTS}

Our objective is to determine the heat generated in a flashlamp-pumped laser rod. In order to use the measured results, we need to evaluate the relation between variations of wavefronts and the generated heat. In some special cases, when wavefront is expressed in series of Zernike functions, we need to determine the correlation between values of Zernike coefficients and generated heat. 
Most of the experimental research on measuring thermal effects in solid laser rod are based on explicit expressions which obtained by W. Koechner [8]. To yield these explicit expressions, Koechner has assumed that the generated heat is uniformly distributed within laser rod. Based on theory of heat transfer in solid state material, Koechner has proved that heat distribution in thermal equilibrium state has the shape of parabolic curve which centered at symmetric axis and approaches zero on either sides, which means that the highest temperature points locate on the symmetric axis of the laser rod. Temperature gradient causes intense local heating that induces a refractive index gradient. As a result, the beam travelling through the laser rod undergoes the same effects as of a convex lens. The explicit expressions obtained by Koechner allow us to determine the dependency of focal length of thermal lens on generated heat density in laser rod. The Koechner's assumptions may lead to rather simple explicit expressions, however, these assumptions are rarely applied to optical pumped solid laser in effect because to achieve uniform heat distribution in laser rod, we need to use some special techniques. In practical application, the heat source distribution is so complicated to be described by explicit expressions. On the other hand, even in the case of high repetition rate solid laser, the pumping pulse width is significantly shorter than the time interval between two pulses. According to solid laser calculation models, pumping process is often considered to be adiabatic [1], which means that the role of heat transfer and dissipation is almost insignificant. Heat transfer and dissipation occur within time interval between two pulses. This is a practical and reasonable assumption. Because pumping process is adiabatic, temperature gradient is generated in laser rod due to pumping process rather than to heat transfer and dissipation process. Temperature gradient in laser rod is caused by heterogeneous heat distribution and heat accumulation during pumping process. For this reason, the variations caused by thermal effects do not depend on heat power, they depend on thermal energy and its distribution in laser rod, which means that it depends on the distribution of absorbed energy. The laser rod may focus (when heat is distributed mainly on the symmetric axis) or diverge (when heat is distributed on the edge) the beam.

Because explicit expressions cannot be derived for absorbed energy distribution, the relation between measured results and generated thermal energy of each quantron configuration can only be obtained from calculation models and numerical methods.

The process of building calculation model for quantron in flashlamp-pumped Nd:YAG solid-state laser using Monte - Carlo ray tracing method with Zemax software is presented in details in $[9,10]$. Using this calculation model, we can rebuild wavefront of the output-collimated beam after it travels through a pumped laser rod using finite element method for each quantron configuration. As for illustration, we show the calculations of 3 quantron configurations whose reflector chamber has cylinder, ellipsoid and oval shape. The parameters of laser rod, flash lamp, cooling liquid system, distance between flash lamp and laser rod, reflective index of reflector chamber are the same for all experiments. The length of major axis and minor axis of the ellipsoid correspondingly are $18.356 \mathrm{~mm}$ and $21.0 \mathrm{~mm}$. The center of flash lamps and center of laser rod locate at the foci of the ellipsoid. The radius of two halves of the oval is $6.0 \mathrm{~mm}$. The distance between centers of two halves of the oval is $9.0 \mathrm{~mm}$. The center of laser rod and center of the flash lamp locate on the center of two halves of the circle. Zernike coefficient is calculated based on the obtained wavefront. In order to calculate Zernike coefficient, we use some Matlab functions coded by S. Carey [11]. The resolution of these calculations corresponds to the resolution of wavefront sensor (the cross section is $4.0 \mathrm{~mm} \times 4.0 \mathrm{~mm}$, the number of microlenses is $29 \times 29$ ). The 
results proved that values of Zernike coefficient depend linearly on generated thermal energy in laser rod. Fig. 4 demonstrates the dependency of Zernike coefficient $C_{2}^{0}$ (according to OSA/ANSI standard [12], which corresponds to the index of 5) on generated thermal energy. The coefficients $C_{2}^{0}$ are calculated in $\mu \mathrm{m}$ and energy is calculated in $\mathrm{J}$. The coefficients $C_{2}^{0}$ are considered critical because it allows to evaluate the impacts of laser rod as a lens. This coefficient is positive when laser rod acts as a concave lens and is negative when laser rod acts as a convex lens. In general cases, the relation between generated thermal energy within laser rod and Zernike coefficient $C_{2}^{0}$ can be expressed as:

$$
E_{h}=\alpha \cdot C_{2}^{0}
$$

where $\alpha$ is rate coefficient which depends on specific quantron configurations. The relation between Zernike coefficient $C_{2}^{0}$ and focal length $f$ is determined by calculating Zernike coefficient $C_{2}^{0}$ from the expression [7]:

$$
f=\frac{r_{0}^{2}}{4 \sqrt{3} C_{2}^{0}}
$$

in which, $r_{0}$ is radius of the pupil of wavefront sensor.

Some other experimental methods can only determine $f$ rather than rebuild wavefront. For this reason, the Zernike coefficient $C_{2}^{0}$ is specially concerned in thermal effects investigations.

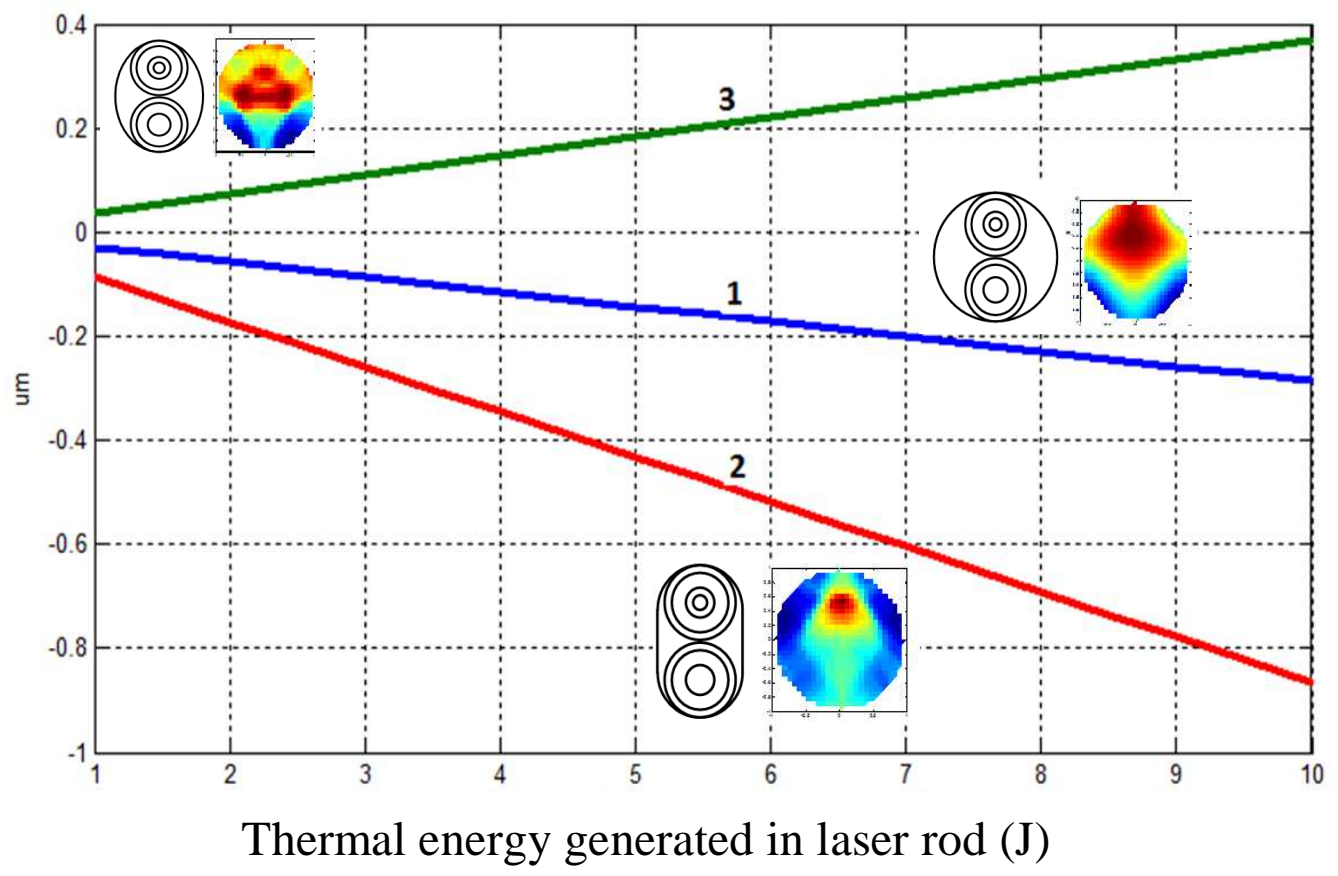

Fig. 4. Dependence of Zernike coefficient $C_{2}^{0}$ on generated thermal energy and its distribution within laser rod.

Fig. 4 shows that in the case of using ellipsoid reflector chamber, the laser rod acts as a convex lens due to thermal effects while in the case of using spherical and oval reflector chamber, 
the laser rod acts as a concave lens. The focusing power of laser rod in the case of using oval reflector is greater than that of the case using spherical reflector. The rate of generated thermal energy $E_{h}$ to Zernike coefficient $C_{2}^{0}$ must be calculated for each specific quantron configuration. In the case of using circle quantron (as illustrated in Fig. 1), from the calculation model, we achieve the results in the Table 1:

Table 1. Dependence of Zernike coefficient $C_{2}^{0}$ on generated thermal energy $E_{h}$.

\begin{tabular}{|c|c|c|c|c|c|c|c|c|c|c|}
\hline$E_{h}(\mathrm{~J})$ & 1 & 2 & 3 & 4 & 5 & 6 & 7 & 8 & 9 & 10 \\
\hline$C_{2}^{0}(\mu \mathrm{m})$ & -0.0287 & -0.0573 & -0.0860 & -0.1146 & -0.1433 & -0.1719 & -0.2006 & -0.2292 & -0.2579 & -0.2866 \\
\hline
\end{tabular}

Based on the obtained values in the Table 1, the rate coefficient $\alpha$ in expression (1) is calculated to be -34.965 . For example, in case of the measured value of $C_{2}^{0}$ is $0.182 \mu \mathrm{m}$, the generated thermal energy $E_{h}$ is calculated to be $6.3636 \mathrm{~J}$.

The resolution (the number of microlenses in a unit area) of wavefront sensor may have profoundly impact on the rebuilt wavefront and hence it affects remarkably on the calculated Zernike coefficients.

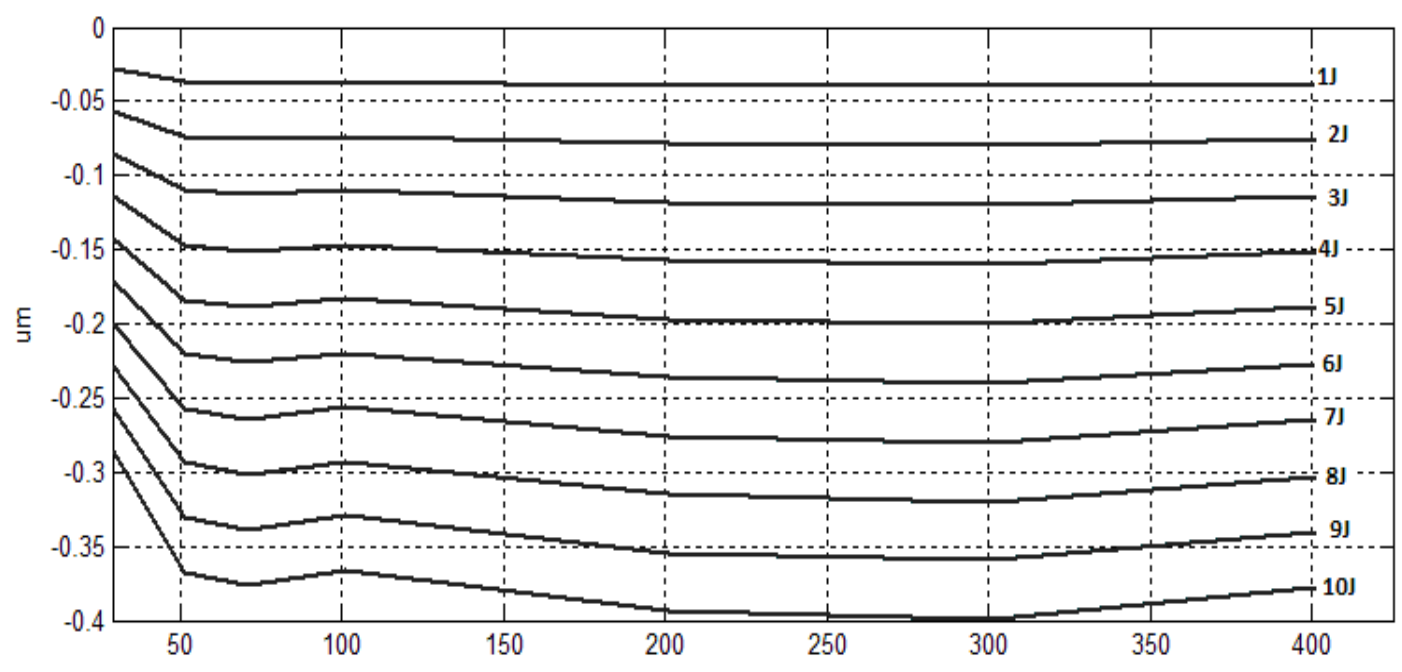

The number of microlenses in a 4-mm long row of wavefront sensor

Fig. 5. Dependence of Zernike coefficients $C_{2}^{0}$ on the resolution and generated thermal energy.

Fig. 5 shows the results of $C_{2}^{0}$ coefficients against changes in the number of microlenses (area of each is $4.0 \mathrm{~mm} \times 4.0 \mathrm{~mm}$ ) in a row with different generated thermal energy (from 1 to $10 \mathrm{~J}$ ) in the laser rod. These results proved that Zernike coefficients become stable when we use several hundreds of microlenses in a row. Clearly the more heat energy is generated, the more distinctive the Zernike coefficients are. For typical commercial Shack-Hartmann sensors at present, the number of microlenses per mm rarely exceeds 10-20, calculation for each specific sensor are critical to analyze the measured results. 


\section{CONCLUSIONS}

Calculation models and numerical methods play an important role in determining the relation between experimental results and generated thermal energy when the distribution of absorbed energy in laser rod is complicated and cannot be described by explicit expressions. The calculation must be processed for each specific quantron configuration and wavefront sensor.

\section{REFERENCES}

[1] A. V. Mezenov, L. N. Soms, A. I. Stepanov, Mechanical engineering, Leningrad (1986) 199 (in Russian)

[2] P. V. Thinh, L. N. Anh, L. V. Dai, Journal of Military Science and Technology 8 (2013) 34 - 40.

[3] A. A. Solviev, Dissertation, Nizhny Novgorod (2002) (in Russian)

[4] S. L. Mikerin, I.G Pantrikova, V.D. Ugozaev, Computer Optics 26 (2004) 37-47 (in Russian)

[5] S. Chenais, F. Druon, F. Balembois, G. Lucas-Leclin, Y. Fichot, P. Georges, R. Gaume, B. Viana, G. P. Aka, D. Vivien, Opt. Mat. 22 (2003) 129-137.

[6] D. Stuéinskas, R. Antipenkov, A. Varanavicius, Lithuanian Journal of Physics 50 (2010) 191-199.

[7] . J. H. Sung, T.M. Jeong, S. K. Lee, T. J. Yu, I. W. Choi, J. Lee, Journal of the Korean Physical Society 55 (2009) 495-500.

[8] W. Koechner, Appl. Opt. 9 (1970) 2548 - 2553.

[9] P. V. Thinh, L. N. Anh, L. V. Dai, Journal of Military Science and Technology 8 (2013) 24 - 27.

[10] P. V. Thinh, L. N. Anh, L. V. Dai, Journal of Military Science and Technology 8 (2013) 28 - 33.

[11] http://www. Mathworks.com/matlabcentral/fileexchange/28665 - zernike3.

[12] American National Standards Institute: Methods for Reporting Optical Aberrations of Eyes (ANSI Z80.28-2004, 2004). 